\title{
A Novel Routing Strategy Towards Achieving Ultra-Low End-to-End Latency in 6G Networks
}

\author{
Satya R. Das ${ }^{1}$, Sayan Sen Sarma ${ }^{2}$, Mitrabinda Khuntia ${ }^{1}$, Indranil Roy ${ }^{3}$, \\ Koushik Sinha ${ }^{3}$, and Bhabani P. Sinha ${ }^{1}$ \\ ${ }^{1}$ Dept. of Computer Science \& Engg., SOA University, \\ Bhubaneswar, India. \\ ${ }^{2}$ Dept. of Computer Science \& Engg., University of Calcutta, \\ West Bengal, India. \\ ${ }^{3}$ School of Computing, Southern Illinois University, \\ Carbondale, USA.
}

\begin{abstract}
Compared to 5G, 6G networks will demand even more ambitious reduction in endto-end latency for packet communication. Recent attempts at breaking the barrier of end-to-end millisecond latencies have focused on re-engineering networks using a hybrid approach consisting of an optical-fiber based backbone network architecture coupled with high-speed wireless networks to connect end-devices to the backbone network. In our approach, a wide area network (WAN) is considered with a high-speed optical fiber grid network as its backbone. After messages from a source node enter the backbone network through a local wireless network, these are delivered very fast to an access point in the backbone network closest to the destination node, followed by its transfer to the local wireless network for delivery to the destination node. We propose a novel routing strategy which is based on distributing the messages in the network in such a way that the average queuing delay of the messages through the backbone network is minimized, and also the route discovery time at each router in the backbone network is drastically reduced. Also, multiple messages destined towards a particular destination router in the backbone network are packed together to form a mailbag, allowing further reductions in processing overheads at intermediate routers and pipelining of mailbag formation and route discovery operations in each router. The performance of the proposed approach green based on these ideas has been theoretically analyzed and then simulated using the ns-3 simulator. Our results show that the average end-to-end latency is less than $380 \mu$ s (with only $46-79 \mu$ s within the backbone network under varying traffic conditions) for a $1 \mathrm{~KB}$ packet size, when using a $500 \mathrm{Gbps}$ optical fiber based backbone network laid over a $15 \mathrm{Km} \times 15 \mathrm{Km}$ area, a $50 \mathrm{Mbps}$ uplink channel from the source to the backbone network, and a 1 Gbps downlink channel from the backbone network to the destination. The significant reduction in end-to-end latency as compared to existing routing solutions clearly demonstrates the potential of our proposed routing strategy for meeting the ultra-low latency requirements of current $5 \mathrm{G}$ and future $6 \mathrm{G}$ networks, particularly for mobile edge computing (MEC) application scenarios.
\end{abstract}

Keywords: 6G, Beyond 5G,5G, Routing, Ultra-low latency, Network architecture, Queuing delay.

DOI: $10.5121 /$ ijcnc.2022.14101 


\section{Introduction}

Recent phenomenal advances in communication networks lead us to the concept of having a hyper-connected world [7] which would eventually involve sharing practically an unlimited amount of data through ubiquitous access by every human being or any device from anywhere around the globe at any time. It has been predicted that in the next few years, more than 30 billion devices will be connected in such a network forming what is called an Internet of Things (IoT) or Internet of Everything (IoE) $[9,6]$, which would imply that more than six devices per person will be actively involved for man-machine interaction [9]. The goal of next generation mobile networks such as $5 \mathrm{G}$ and $6 \mathrm{G}$ networks is to interconnect such wireless devices through both cellular networks and WiFi as well as through the use of ad hoc links, such as those found in wireless sensor networks and VANETs. The overall objective of such networking is to create a platform to provide various types of complex services in a smart world ranging from efficient transportation in a city, efficient distribution and usage of energy and water, household device control, industrial process control to providing medical facilities to citizens, homeland security, defense, etc. [4].

Compared to currently existing $4 \mathrm{G}$, the goals set for the $5 \mathrm{G}$ network communication included: i) handling thousand times higher volume of data traffic, ii) providing ten times higher data rate, iii) reducing end-to-end latency for packet communication by five times, iv) increasing battery life by ten times, and v) increased security. As recommended by the International Telecommunication Union (ITU), the three major areas of $5 \mathrm{G}$ which show great promise for future growth are enhanced mobile broadband (eMBB), ultra-reliable low-latency communications (URLLC), and massive machine-type communications (mMTC) [19]. Quality of service (QoS) requirements for these include end-to-end (E2E) secure and reliable delivery of data with ultra-low latencies [26].

As such, it is estimated that by $2030,5 \mathrm{G}$ will reach its limits $[11,25]$ in terms of its ability to cater to the bandwidth and QoS demands. Driven by such projections, research and standardization efforts of $6 \mathrm{G}$ wireless networks for a hyper-connected society beyond 2030 has been initiated across the globe by the ITU. From a survey of research papers on $6 \mathrm{G}$ networks $[29,11,28,3,8,24,20]$, we summarize below the basic properties of $6 \mathrm{G}$ networks that are of interest to us.

- Peak Rate (Terabit Era): Based on our experience about the increase in date rate from $1 \mathrm{G}$ to $5 \mathrm{G}$ communication systems, ITU [20] has predicted that the peak demand in next 10 years (by 2030) will be about 1 Terabit/s (Tbps).

- Higher Energy Efficiency: In order to achieve extremely high throughput and channel bandwidth while supporting significantly larger number of wireless nodes, $6 \mathrm{G}$ networks will face the daunting challenge of addressing the large amounts of energy required for such communication. Hence, reduction of en- 
ergy consumption per bit (J/bit) as much as possible will be one of the main criteria of $6 \mathrm{G}$ networks $[29,11]$.

- Connection Everywhere and Anytime: The communication goal of $6 \mathrm{G}$ is to provide a network connectivity so as to facilitate ubiquitous communication and interaction between people and objects, anywhere and any time [29,28].

- Self-Aggregating Communications Fabric: 6G should be capable of dynamically integrating a variety of technology systems, including self-aggregation ability with different types of network architectures [8].

- Ultra-low Latencies: 6G should be able to provide extremely low-latency communications (user-plane latency of about $100 \mu \mathrm{s}$, control-plane latency of about $1 \mathrm{~ms}$, and processing delay of about 10ns [3,24]) in order to satisfy QoS/Quality of Experience (QoE) requirements of future real-time applications. Fig. 1 provides an overview of average end-to-end latency tolerances in several $5 \mathrm{G}$ and future $6 \mathrm{G}$ network applications.

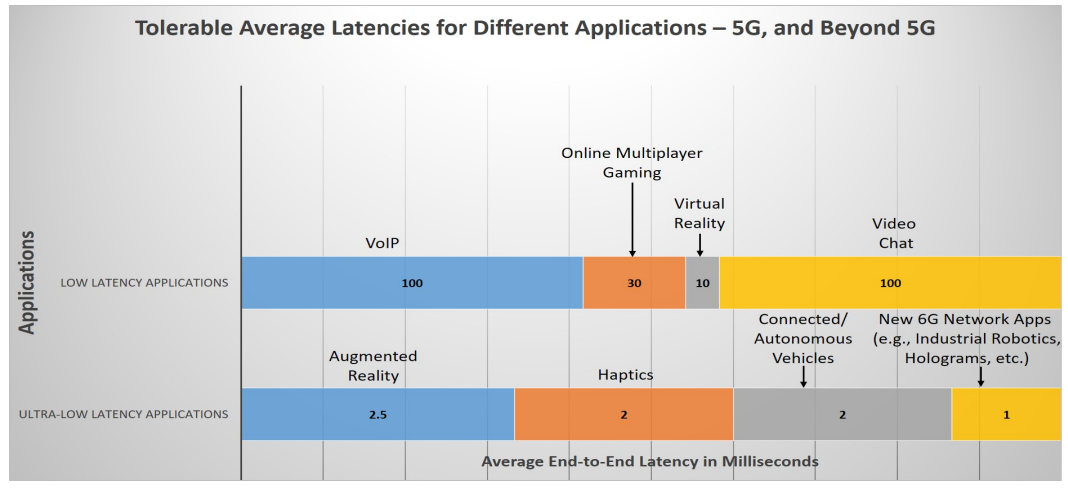

Fig. 1: Comparison of average end-to-end latency tolerance in various applications

We specifically focus here on the aspect of reducing the end-to-end packet communication time in a wide area network (WAN) which finds potential application in ultra-low latency based mobile edge computing (MEC) environments [30, 22, 2]. Routing techniques with minimum control overhead and bandwidth for reducing the route discovery time and end-to-end packet transmission latency have been proposed in $[13,5]$. A number of ideas have also been proposed for theoretical description of Internet routing and their real life implementations. Routing Information Protocol (RIP) [16], Open Shortest Path First (OSPF) [17, 12], Border Gateway Protocol (BGP) [21] etc. are the significant ones among them. Software-Defined Networking (SDN) [14] is another approach for managing the real-time performance criteria of message communication in dynamic networks. SDNs accomplish 
this by dissociating the forwarding process of network packets (data plane) from the routing process (control plane).

Tariq et al. [24] compared the probable improvements in the key performance indicators (KPI) for $6 \mathrm{G}$ networks vis-à-vis $5 \mathrm{G}$ networks as shown in Table 1, where the latency for U-plane and C-plane refer to the latency for user-plane and controlplane, respectively. It appears that none of the existing techniques would be able to satisfy the targeted reduction of end-to-end latency and hence, some completely different appropriate routing strategy in achieving this goal of reduced packet communication time is urgently called for to implement the 6G standard.

In a recent paper [18], a new hybrid fiber-optic-wireless based architecture that uses a combination of multiple interconnected fiber-optic ring based network of edge nodes, and wireless networks connecting end-devices such as user equipments (UEs) to such a fiber-optic ring system, has been proposed for achieving latencies below $1 \mathrm{~ms}$ to address the future requirements of $6 \mathrm{G}$ networks. However, [18] neither proposed a routing scheme nor provided an analysis of end-to-end latency in their proposed fiber-optic-wireless ring system.

\section{Our Contribution:}

We propose here a novel routing strategy to achieve ultra-low latency for communication in a $6 \mathrm{G}$ wide area network (WAN). Our approach is based on using a hybrid optical-wireless architecture for the WAN which will have a backbone grid network constituted by high-speed optical fibers, along with local wireless network access points. Messages from a source node are routed to the nearest access point on this backbone grid network through the local wireless network. The backbone network then delivers the message to some access point nearest to the destination node with sub-millisecond level latency. Finally the message reaches its desired destination from this access point through a local wireless network. Every intersection point of the backbone grid network will have a router which will have the ability to route a message along right, left, up or down from that intersection point of the grid. In order to significantly reduce the message routing overhead in intermediate routers, multiple messages from a source point destined to the same final destination point in the backbone network are grouped together to form a mailbag. Next, a novel technique of routing this mailbag to its desired destination through one of the shortest paths in the grid network is devised so that the average queuing delay at the intermediate routers on the backbone network is minimized, and at the same time the route discovery time at each router in the backbone network is drastically reduced. Further, the size of the mailbag is judiciously chosen so that the time for transferring the mailbag data to the optical fiber at each source point (router) in the backbone network matches with the route discovery time, to enable pipelining of data transfer and route discovery operations in each router. All these facts eventually lead to achieving a much reduced end-to-end latency for message communication. Simulation of our proposed approach using ns-3 shows that end- 
to-end latency for a packet of size $1 \mathrm{~KB}$ is, on an average, less than $380 \mu \mathrm{s}$ using a 500 Gbps optical fiber grid network as the backbone laid over a $15 \mathrm{Km} \times 15$ Km city area and a wireless communication channel having 50 Mbps uplink speed from the source and $1000 \mathrm{Mbps}$ downlink speed to the destination. Our simulation results also show that out of the $380 \mu$ s average end-to-end latency, routing the packet through the backbone network requires only $46-79 \mu$ s under varying traffic conditions, while the rest of the time is being consumed by the local wireless networks.

Given the observed end-to-end latencies in our ns-3 simulations, we believe that our proposed approach for reducing average end-to-end latency to sub-millisecond levels is highly promising for both $5 \mathrm{G}$ and $6 \mathrm{G}$ networks, especially for delivering MEC based solutions over a WAN.

Table 1: Key performance indicators for $5 \mathrm{G}$ and $6 \mathrm{G}$ networks

\begin{tabular}{|c|c|c|c|}
\hline \multicolumn{2}{|c|}{ Characteristics } & $5 \mathrm{G}$ & $6 \mathrm{G}$ \\
\hline \multicolumn{2}{|c|}{ Operating frequency } & $3-300 \mathrm{GHz}$ & up to $1 \mathrm{THz}$ \\
\hline \multicolumn{2}{|c|}{ Data rate (DL) } & 20 Gbps & $>1$ Tbps \\
\hline \multicolumn{2}{|c|}{ Individual data rate } & $1 \mathrm{Gbps}$ & $100 \mathrm{Gbps}$ \\
\hline \multirow{2}{*}{ Latency } & U-plane & $0.5 \mathrm{~ms}$ & $<0.1 \mathrm{~ms}$ \\
\hline & C-plane & $10 \mathrm{~ms}$ & $<1 \mathrm{~ms}$ \\
\hline \multicolumn{2}{|c|}{ Mobility } & up to $500 \mathrm{~km} / \mathrm{hr}$ & up to $1000 \mathrm{~km} / \mathrm{hr}$ \\
\hline
\end{tabular}

\section{Some Preliminaries}

\subsection{Current Scenario of Communication through Optical Fibers}

In a research result of Technical University (TU) Munich, published in 2016 [1], it was reported that Nokia Bell Labs, Deutsche Telekom T-Labs and TU Munich performed a field trial over an optical fiber network deployed by Deutsche Telekom. The field trials reported the successful achievement of one Tbit/s (Terabit/s) in net transmission rate. Their experiment used a modulation technique based on Probabilistic Constellation Shaping (PCS) [10]. In March 2019, it was reported in [1] that an inter-city field experiment between Munich and Regensburg for data transmission via fiber optic cable under real-world conditions was conducted by Nokia and M-Net. The experiment was able to achieve a transfer speed up to 500 Gbit/s over a single wavelength.

Typically a fiber optic cable consists of thousands of strands and each signal uses only one wavelength on a fibre strand. However, since an individual signal does not need to utilize the full spectrum carried by the fiber, every fiber strand can be 
used to provide more than 100 channels. Thus, assuming $500 \mathrm{Gbit} / \mathrm{s}$ transfer rate for each wavelength [1], an overall transmission capacity of more than $50 \mathrm{Tbit} / \mathrm{s}$ can be achieved.

\subsection{Proposed Routing Model}

Similar to the concept of a hybrid fiber optic-wireless (Fi-Wi) architecture presented in [18], we also use here a hybrid FiWi architecture. But in contrast to the multiple rings in the backbone network proposed in [18], a Manhattan grid network of optical fibers as shown in Fig. 2 is used here to constitute the backbone. This optical fiber backbone network will be easily accessible from every user terminal (UT) through an appropriate wireless network (e.g., cellular or WiFi) depending on the type of $U T$. Message packets generated from a source $S$ will be brought to its nearest intersection point (grid point) of the backbone network through a wireless channel of low/moderate speed. Similarly, all message packets meant for the destination node $D$ will be taken out from its nearest grid point of the backbone network, to finally reach $D$ through a wireless channel of low/moderate speed. Thus, the first and last legs of the route are through wireless channels and the intermediate ones are through the backbone optical network. In our later discussions, the terms grid point and intersection point will be used interchangeably. This hybrid Fi-Wi network contains routers at each intersection point of the grid for routing the message along any of the four possible directions - left/right/up/down. Each router will have the

fiber media converters for optical to optical, optical to electrical and electrical to optical conversion to facilitate the mixed fiber-optic-wireless communication [18, $27,15]$.

We can visualize this arrangement as a mail transportation system in which at any grid point, messages received from different neighboring sources, all destined towards a particular destination grid point, will be packed together to form a mailbag. From any intermediate router (located at the grid points), each such mailbag can be routed through the horizontal (rightward/leftward) or vertical (upward/downward) optical links to finally reach its desired destination on the backbone network.

At every intersection point, two buffers will be used, each in the form of a queue - a transmit buffer and a receive buffer. The transmit buffer will be used to store all the mailbags to be forwarded to the next intersection point on the grid network, while the receive buffer will store the mailbags received from other intersection points from which the individual packets will be delivered to their respective final destination nodes through local wireless channels.

The total time for routing a mailbag from the source to the destination along the network consists of several components as discussed below. 


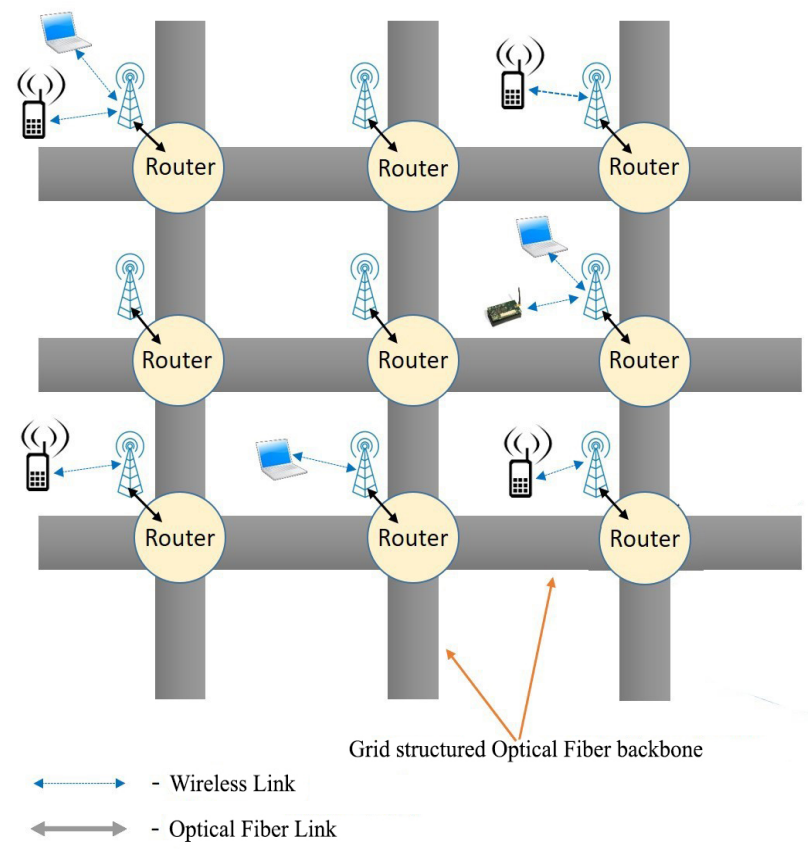

Fig. 2: Optical Fiber-Wireless (Fi-Wi) network architecture for WAN

\section{Routing through Backbone Network}

The total time $T$ required for transmitting a message from a source node $S$ to a destination node $D$ in the backbone grid network can be expressed as,

$$
T=T_{\text {transfer }}+T_{\text {wait }}+T_{\text {prop }}+T_{\text {discover }}
$$

where i) $T_{\text {transfer }}$ is the data transfer time required for transferring the data to a channel with a given capacity (in terms of bit/s), ii) $T_{\text {wait }}$ is the waiting time which will be the sum of waiting times at all intermediate nodes of the grid along the chosen route, iii) $T_{\text {prop }}$ is the propagation time required for message propagation along the links of the grid network, and iv) $T_{\text {discover }}$ is the route discovery time to find a suitable route from $S$ to $D$, possibly with the smallest routing time.

Note that under the assumption of $500 \mathrm{Gbps}$ data transfer rate per wavelength, a packet of size 1000 bytes or $8 K$ bits can be put on the link for transmission in only $8000 /\left(5 \times 10^{11}\right) \mathrm{s}=16 \mathrm{~ns}$. If a mailbag of size, say, $10 \mathrm{~KB}$ is formed at a time by putting 10 small packets each of $1 \mathrm{~KB}$ size, then the transfer time of this mailbag on the optical fibre link at each intersection point is $10 \times 16 \mathrm{~ns}=160 \mathrm{~ns}$.

Considering the fact that the speed of light is approximately 1.5 times less through optical fiber than in vacuum, the time taken by the signal to travel through 
an optical fibre, turns out to be about $5 \mu$ s per kilometer. If each fibre link (the fiber segment between two routers) has a length of $3 \mathrm{~km}$, then the propagation time through this link is $3 \times 5 \mu \mathrm{s}=15 \mu \mathrm{s}$ (approximately).

In order to reduce $T$ by almost an order of magnitude, each of these four components must be reduced as much as possible. In particular, it may be thought that $T_{\text {discover }}$ can be made very small, because we are considering a route between two fixed points $S$ and $D$ in the grid network. However, it may be noted that there exist a large number of possible routes between any two grid points, even with the shortest route length. Specifically, if $n$ is the minimum number of links (counted along both horizontal and vertical directions) to be traversed for reaching $D$ from

$S$, then there are $\frac{1}{n+1} \frac{(2 n) !}{(n !)^{2}}$ possible (shortest) paths of same length $n$ between $S$ and $D$, and this number of possible paths grows exponentially with $n$. In a reallife situation with a dynamically changing traffic load, it is quite likely that there may be heavy congestion at some of the grid points, and a mailbag being routed through such a grid point may suffer a huge queuing delay, making its $T_{\text {wait }}$ very high. Thus, unless the route is judiciously chosen from among such a huge number of possibilities taking the dynamically varying traffic into consideration, $T_{\text {wait }}$ may be very high making the overall scheme to be unacceptable for meeting our goal of reducing end-to-end communication latency. On the other hand, at the same time, the algorithm for selecting such a route to keep $T_{\text {wait }}$ small, must be simple enough so as to have a small value for $T_{\text {discover }}$. These facts make the router design problem quite challenging. In the following sections, our proposed technique is described for keeping both $T_{\text {discover }}$ and $T_{\text {wait }}$ small enough to meet our desired objective.

\subsection{Queuing Delay Optimization}

In this section, the queuing delay at the intersection points is analyzed so as to find the condition to minimize the value of $T_{\text {wait }}$. However, to start with, we make the following assumptions :

- i) The Manhattan grid is a square of size $M \times M$.

- ii) A single destination located at the topmost and rightmost corner of the grid network.

- iii) Every intersection point can be a source of new mailbag generation. The pattern of new mailbag generation at any intersection point $(i, j)$ follows Poisson distribution with a mean generation rate of $\lambda_{i j}$ per second.

- iv) The service rate of a mailbag is given by $\mu=\frac{1}{T_{\text {transfer }}}$. It will be assumed that at any intersection point $(i, j), 1 \leq i, j \leq M$, this $\mu$ value is same and is greater than the total arrival rate of mailbags at $(i, j)$ which includes the new mailbags generated at $(i, j)$ and those coming from other neighboring intersection points. 
Conditions i) and ii) above will be relaxed later to consider a rectangular grid structure and also the destination point to be any point other than the topmost rightmost corner point of the grid, respectively.

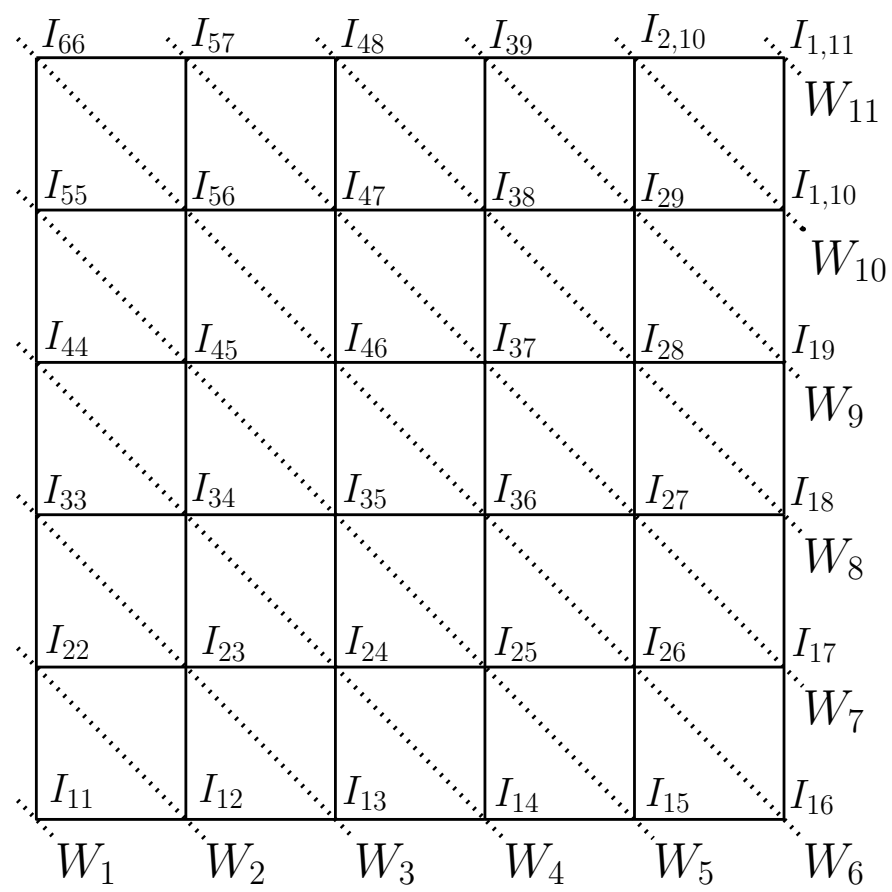

Fig. 3: A $6 \times 6$ grid network with transmission fronts $W_{1}, W_{2}, \cdots, W_{11}$ with respect to $I_{1,11}$ as the single destination point

At this point, to estimate the optimal value of the average queuing delay, we follow the technique similar to that used by Sarma et al. [23] for minimizing traffic delay in Manhattan grid-structured road networks. Assume that the upper-right corner of the grid is the only destination point of all messages. Next, draw an imaginary line connecting the bottommost leftmost intersection point of the grid the topmost rightmost corner of the grid. Then draw a set of parallel lines, each perpendicular to this imaginary line and passing through the grid points, which are termed as transmission-fronts. Fig. 3 shows an example of such transmission fronts $W_{1}, W_{2}, \cdots, W_{11}$ of a $6 \times 6$ grid with respect to the topmost rightmost intersection point as the single destination. The numbering scheme of the intersection points $I_{i j}$ 's on the transmission-front $W_{j}, j=1,2, \cdots, 11$ is shown in Fig. 3 with $I_{11}$ on $W_{1}$ as the bottommost leftmost intersection point and $I_{1,11}$ on $W_{11}$ as the topmost rightmost intersection point. It may be noted that the Manhattan distance of all intersection points on a given transmission-front $W_{j}, j=1,2, \cdots$ from the bottom- 
most leftmost intersection point is always the same. Thus, these transmission-fronts may be considered as similar to the wave-fronts in a wave propagation system. Total number of intersection points $I_{i j}$ 's over a transmission-front $W_{j}$ is given by,

$$
P_{j}= \begin{cases}j, & \text { for } 1 \leq j \leq M \\ 2 M-j, & \text { for } M<j \leq 2 M-1\end{cases}
$$

Let $f_{i j}$ be the fraction of the total number of mailbags diverted from the intersection point $I_{i j}$ on $W_{j}$ towards right to the intersection point $I_{i, j+1}$ (when it exists) on $W_{j+1}$. Hence, the remaining $1-f_{i j}$ fraction will be transmitted along the vertical link (when it exists) from $I_{i j}$ to the intersection point $I_{i+1, j+1}$ on $W_{j+1}$. All these mailbags arriving at the intersection points $I_{i, j+1}$ and $I_{i+1, j+1}$ on $W_{j+1}$ from $W_{j}$ will add to the new mailbags $\lambda_{i, j+1}$ and $\lambda_{i+1, j+1}$ generated at $I_{i, j+1}$ and $I_{i+1, j+1}$, respectively. The total number of mailbags at any intersection point $I_{i, j+1}$ after this process is denoted as $\lambda_{i, j+1}^{\prime}$.

Authors in [23], solved a similar optimization problem to minimize the traffic delay in Manhattan road networks which leads to the following lemma:

Lemma 1. To achieve minimum average queuing delay at a transmission-front $W_{j+1}$, the mailbags to be transmitted from $W_{j+1}$ should be evenly distributed over all the intersection points on it.

\subsection{Optimal Load Distribution}

To minimize the queuing delay at the transmission-front $W_{j+1}$ following the results of Lemma 1, we have to derive the optimal load distribution fractions $f_{i j}$ 's at all the intersection points on $W_{j}$ for routing the traffic to the inon $\lambda_{1, j+1}^{\prime}=\lambda_{2, j+1}^{\prime}=$ $\cdots=\lambda_{j+1, j+1}^{\prime}$. It may be noted that $\Delta_{i, j+1}$ monotonically increases with $\lambda_{i, j+1}^{\prime}$, and $\forall i, 1 \leq i \leq j+1, \lambda_{i, j+1}^{\prime}$ is a linear function of $f_{i j}$ which monotonically increases with $f_{i j}$ and for $2 \leq i \leq j+1, \lambda_{i, j+1}^{\prime}$ is a monotonically decreasing linear function of $f_{i-1, j}$. A similar situation was also encountered in [23] while distributing the vehicular traffic in an optimal way in a grid network of roads. Thus, we can use the results derived in [23] to find the required optimal values of $f_{i j}$ 's for minimizing the average queuing delay at the transmission-front $W_{j+1}$, as described below.

Case 1: $j<M$ (when the number of intersection points on the transmissionfront $W_{j}$ increases with increase in $j$ ). In this case the optimal values of $f_{i j}$ 's will follow directly from the results given in Theorem 1 of [23].

Case 2: $j \geq M$ (when the number of intersection points on the transmissionfront $W_{j}$ decreases with increasing $j$ ). In this case the optimal values of $f_{i j}$ 's will follow directly from the results given in Theorem 2 of [23].

In a network with a dynamically changing traffic, the $\lambda$-values at different intersection points will be periodically determined from a knowledge of new mailbag generation during an immediate past time interval so as to compute the optimal fractions for mailbag distribution following the above results. 


\subsection{Route Discovery Time}

The optimal traffic distribution factor $f_{i j}$ towards the right link at different intersection points (routers) of the grid can be stored in a two-dimensional table. At each intersection point $I_{i j}$, to decide about the correct direction of movement (upward or rightward) of a given mailbag $\mathcal{M}$, the entry $f_{i j}$ from this two-dimensional table is retrieved from a cache memory in a few machine cycles. Simultaneous with retrieving this table entry, a uniform random number, say $r$, in the interval $[0,1]$ is also generated. If $r<f_{i j}$, then $\mathcal{M}$ is routed towards the right link; otherwise $\mathcal{M}$ is routed towards the up link.

Since such a uniform random number generation can be done by executing only a few tens of machine language instructions, assuming a clock cycle time of $1 \mathrm{~ns}$, it can always be completed in a few tens of nanoseconds. Reading the fraction from the cache and formation of the mailbag at that particular intersection point, can be done in parallel with this random number generation. All these can be completed in a time much less than 100 ns. Let us call this time as $t_{c o m p}$ at each intersection point where $t_{\text {comp }} \leq 100 \mathrm{~ns}$.

If a mailbag has to traverse a total of $n$ links to reach the destination from its source, then $T_{\text {discover }}$ reduces to reading a total of $n$ such table entries and $n$ random number generations, i.e., $T_{\text {discover }}=n \times t_{\text {comp }}$.

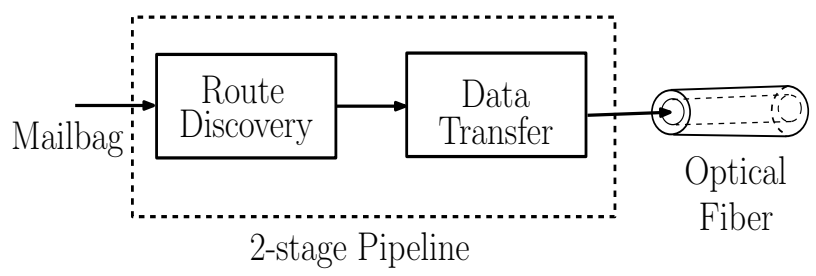

Fig. 4: Pipelined architecture for mailbag transmission

At this point, we propose a pipelined architecture, as shown in Fig. 4, for transferring the mailbags to the horizontal or vertical optical fiber cables at each intersection point. Noting that $T_{\text {transfer }}$ and $T_{\text {discover }}$ both are of the same order of magnitude ( 100 to $160 \mathrm{~ns}$ per intersection point), the overall throughput for endto-end packet communication can be increased by such pipelining. Hence, considering an infinite stream of such mailbags, the maximum time for route discovery, data transfer and data propagation time for each mailbag traversing a Manhattan distance of $30 \mathrm{Km}$ through 10 intersection points will effectively be equal to $10 \times(15+0.16) \mu \mathrm{s}=151.6 \mu \mathrm{s}$. However, the average values of these components of routing time will be smaller than the this maximum value of $151.6 \mu$ s and can be 
estimated by considering the total traffic at each intersection point, as illustrated by the following example.

Example 1. Consider the $6 \times 6$ grid network of Fig. 3. The Manhattan distance in terms of the number of links to be traversed by a mailbag generated at an intersection point $I_{i j}$ to reach the destination point $I_{1,11}$ is equal to $(11-j)$. Assume that the number of mailbags generated per unit time at each intersection point is uniform and is equal to $\lambda$. Hence, the average propagation distance of a mailbag along the grid under this assumption will be equal to $(\lambda .10+2 \lambda .9+3 \lambda .8+\cdots+6 \lambda .5+$ $5 \lambda .4+\cdots+2 \lambda .1) /(\lambda+2 \lambda+\cdots+6 \lambda+5 \lambda+\cdots+2 \lambda) \times 3 \mathrm{Km}=\frac{180}{35} \times 3 \mathrm{Km}=12.67$ $\mathrm{Km}$. Thus, the average propagation time of a mailbag is equal to $5 \times 12.67 \mu \mathrm{s}=$ $63.35 \mu \mathrm{s}$, assuming that the signal propagation time is $5 \mu \mathrm{s}$ per kilometer. Also, the average number of intersection points traversed by a mailbag will be equal to $(\lambda .10+2 \lambda .9+3 \lambda .8+\cdots+6 \lambda .5+5 \lambda .4+\cdots+2 \lambda .1) /(\lambda+2 \lambda+\cdots+6 \lambda+5 \lambda+\cdots+2 \lambda)$ $=\frac{180}{35}=5.14$. Thus, the average time through the pipelined unit of route discovery and data transfer for each mailbag will be equal to $5.14 \times 0.16 \mu \mathrm{s}=0.8224 \mu \mathrm{s}$. Hence, altogether the average value of $T_{\text {discover }}+T_{\text {transfer }}+T_{\text {prop }}$ per mailbag of $10 \mathrm{~KB}$ size comes out to be $0.8224+63.35 \mu \mathrm{s}=64.1724 \mu \mathrm{s}$.

\section{Routing through Wireless Communication Segments}

In the first leg of our proposed communication architecture, packets generated from a source node $S$ will reach its nearest intersection point on the backbone network through wireless communication. Similarly, the last leg of communication involves disseminating the packets from an intersection point of the backbone network to the respective final destination nodes around it. To be more specific, a packet sent from a source node will be stored in the transmit buffer of the nearest intersection point of the grid to be included in a mailbag depending on its destination node $D$. Thus, there is no queuing delay involved in this first leg. In the last leg of the communication system, however, there will be a queuing delay involved in the communication process as the packets from the receive buffer of the intersection point on the grid will be serviced one by one to be transferred to their desired destinations. We assume that the data transfer speed for uplinking is $B_{u}$ bps and that for downlinking is $B_{d}$ bps. Usually $B_{u}<B_{d}$. Various components of routing time involved in these wireless communication segments are now discussed below.

\subsection{Packet Uploading Time}

The data transfer time for uplinking a packet of size $L$ bits is given by $T_{t}^{u}=L / B_{u}$. Assuming that the backbone grid has all links of same length $l$, the maximum distance to be traversed by a packet to reach the nearest intersection point on the grid from a source node is given by $d=\frac{l}{\sqrt{2}}$ and the corresponding propagation 
time $T_{p}^{u}$ is less than or equal to $d / c, c$ being the velocity of electromagnetic waves in free space. As already mentioned above, there is no queuing delay involved in this uploading step.

\subsection{Packet Downloading Time}

In this case, data transfer time for a packet is given by $T_{t}^{d}=L / B_{d}$. The propagation time in this case also is given by $T_{p}^{d} \leq d / c$. However, there will be a queuing delay $T_{q}$ involved here which is given by

$$
T_{q}=\frac{K_{\text {download }}}{1-\lambda_{\text {download }} / \mu_{\text {download }}}
$$

where the constant $K_{\text {download }}$ is the service time required by the queue for transferring one packet of length $L$, and hence, is equal to $L / B_{d}, \mu_{\text {download }}$ is the maximum number of packets that can be serviced per unit time, i.e., $\mu_{\text {download }}$ is

given by $\frac{1}{L / B_{d}}$, and $\lambda_{\text {download }}$ is the average number of packets joining the queue (in the receive buffer) per unit time.

Overall, the total routing time involved in the wireless segments of communication is given by $T_{\text {wireless }}=T_{t}^{u}+T_{t}^{d}+2 T_{p}+T_{q}$.

\section{Estimation of Overall Routing Time}

The end-to-end routing time for our proposed approach can now be estimated, using the derived expressions for latencies in the backbone network, and in the source and destination-side local wireless networks attached to the backbone network.

\subsection{Routing Time through Backbone Network}

To estimate the contribution of the backbone network to the overall routing time, we assume that the data transfer rate along only one strand of the optical fiber cables in horizontal or vertical direction at each intersection point is $0.5 \times 10^{12} \mathrm{bps}$. Thus, the service rate $\mu$ can be taken as equal to $0.5 \times 10^{12} \mathrm{bps}$, i.e., $\frac{1}{\mu}=2 \times 10^{-12}$ s. On the other hand, the service time (data transfer time) for a mailbag containing 10 packets each of size $1 \mathrm{~KB}$, i.e., $8 \times 10 \times 10^{3}=8 \times 10^{4}$ bits will be equal to $16 \times 10^{-8}$ s. The above value of $\mu=0.5 \times 10^{12}$ can be normalized to be equal to 1 , so that arrival of 1 million mailbags per second at an intersection point will be equivalent to an effective $\lambda$ value of $10^{6} \times 8 \times 10^{4} \times 2 \times 10^{-12}=0.16$.

Example 2. Consider again the $6 \times 6$ grid network as shown in Fig. 3. We assume that the $6 \times 6$ grid has fixed mailbag generation rates over its intersection points as given below: $\lambda_{11}=0.05, \lambda_{12}=0.03, \lambda_{22}=0.02, \lambda_{13}=0.01, \lambda_{23}=0.03, \lambda_{33}=0.04$, $\lambda_{14}=0.05, \lambda_{24}=0.04, \lambda_{34}=0.02, \lambda_{44}=0.06, \lambda_{15}=0.03, \lambda_{25}=0.02, \lambda_{35}=0.03$, 
$\lambda_{45}=0.04, \lambda_{55}=0.02, \lambda_{16}=0.02, \lambda_{26}=0.02, \lambda_{36}=0.02, \lambda_{46}=0.02, \lambda_{56}=0.03$, $\lambda_{66}=0.02, \lambda_{17}=0.01, \lambda_{27}=0.02, \lambda_{37}=0.04, \lambda_{47}=0.01, \lambda_{57}=0.02, \lambda_{18}=0.02$, $\lambda_{28}=0.03, \lambda_{38}=0.02, \lambda_{48}=0.02, \lambda_{19}=0.03, \lambda_{29}=0.03, \lambda_{39}=0.01, \lambda_{1,10}=0.04$, $\lambda_{2,10}=0.05$.

Considering the intersection point $I_{1,11}$ as the destination point, the total average queueing delay from $W_{1}$ to $W_{11}$ can be calculated as $45.57894 K$. Thus, the average value of $T_{\text {wait }}$ is less than $46 \times 160 \mathrm{~ns}=7.36 \mu$ s (assuming $K=160 \mathrm{~ns}$ with only one strand of the optical fiber cable used for communication).

For the traffic generated at various intersection points as given above, the average number of intersection points traversed by a mailbag can be computed as $\Sigma_{i, j} \lambda_{i j} \times d_{i j}$ over all possible values of $i$ and $j$, where $d_{i j}$ is the rectilinear distance of the destination point from $I_{i j}$ (in terms of the number of links) along the shortest route. This value is computed as 5.33 .

Thus, the average value of $T_{\text {discover }}+T_{\text {transfer }}$ is equal to $5.33 \times 0.16 \mu \mathrm{s}=0.8528$ $\mu \mathrm{s}$. Similarly, the average value of $T_{\text {prop }}$ is found to be equal to $15 \times 5.33 \mu \mathrm{s}=79.95$ $\mu \mathrm{s}$.

Adding all these components, the overall average routing time per mailbag through the optical backbone network for the above example values of traffic generation rates is equal to $T_{\text {discover }}+T_{\text {transfer }}+T_{\text {prop }}+T_{\text {wait }}=(0.8528+79.95+7.36)$ $\mu \mathrm{s}=88.1628 \mu \mathrm{s}$.

It is worth noting that $T_{\text {prop }}$ is the major part (about $90 \%$ ) of this routing time, the component $T_{\text {discover }}+T_{\text {transfer }}$ is very insignificant (only $0.8528 \mu \mathrm{s}$, i.e., $1 \%$ of the total routing time) and the component $T_{\text {wait }}$, i.e., total queuing delay at all intermediate routers is quite small (only $7.36 \mu \mathrm{s}$ ) accounting for $10 \%$ of the overall routing time.

\subsection{Routing Time through Wireless Link Segments}

We now estimate the contribution of the first and last legs of communication, i.e., the wireless communication links to the overall routing time. Commercially available standard home uplinking speeds usually vary from $10 \mathrm{Mbps}$ to $50 \mathrm{Mbps}$. Assuming a packet size of $1 \mathrm{~KB}$, data transfer time $T_{t}^{u}$ for uploading comes out to be $0.8 \mathrm{~ms}, 0.4 \mathrm{~ms}, 0.27 \mathrm{~ms}$ and $0.16 \mathrm{~ms}$ for uplink speeds of $10 \mathrm{Mbps}, 20 \mathrm{Mbps}, 30$ Mbps and $50 \mathrm{Mbps}$, respectively. Assuming the length $l$ of each link of the backbone grid network as $3 \mathrm{Km}$, the propagation time $T_{p}$ comes out to be always less than $2.4 /\left(3 \times 10^{5}\right) \mathrm{s}=8 \mu \mathrm{s}$. In a real life scenario, it may not always be possible to form the layout of a backbone grid network having all links of equal length due to some topographical constraints. However, this assumption is made here only for ease of computation.

For the downloading part at the last leg of communication, we assume the commercially available standard values of $100 \mathrm{Mbps}$ to 1 Gbps as the downlink 
speed of data transfer. Thus, data transfer time $T_{t}^{d}$ comes out to be $80 \mu \mathrm{s}, 40 \mu \mathrm{s}$, $16 \mu \mathrm{s}$ and $8 \mu \mathrm{s}$, for downlink speeds of $100 \mathrm{Mbps}, 200 \mathrm{Mbps}, 500 \mathrm{Mbps}$ and $1 \mathrm{Gbps}$, respectively.

The value of $K_{\text {download }}$ in eq. (2) assumes the value of $80 \mu \mathrm{s}, 40 \mu \mathrm{s}, 16 \mu \mathrm{s}$ and 8 $\mu \mathrm{s}$, for downlink speeds of $100 \mathrm{Mbps}, 200 \mathrm{Mbps}, 500 \mathrm{Mbps}$ and 1 Gbps, respectively. Assuming a value of 0.8 for the fraction $\lambda_{\text {download }} / \mu_{\text {download }}$, the value of queuing delay $T_{q}$ turns out to be $400 \mu \mathrm{s}, 200 \mu \mathrm{s}, 80 \mu \mathrm{s}$ and $40 \mu \mathrm{s}$ for data downlink speeds of $100 \mathrm{Mbps}, 200 \mathrm{Mbps}, 500 \mathrm{Mbps}$ and $1 \mathrm{Gbps}$, respectively.

The values of $T_{\text {wireless }}=T_{t}^{u}+T_{t}^{d}+2 T_{p}+T_{q}$ obtained from these estimates for $\lambda_{\text {download }} / \mu_{\text {download }}=0.8$ are shown in Table 2 for different combinations of uplink and downlink data transfer speeds. From this table, we see that for uplink speed of $50 \mathrm{Mbps}$ and downlink speed of $1 \mathrm{Gbps}, T_{\text {wireless }}=224 \mu \mathrm{s}$. At this downlink speed of $1 \mathrm{Gbps}, \frac{1}{\mu_{\text {download }}}=8 \mu \mathrm{s}$, i.e., $\mu_{\text {download }}=125,000$ packets per second. Thus, $\lambda_{\text {download }} / \mu_{\text {download }}=0.8$ at this speed implies an average arrival rate of 100,000 packets per second which are distributed from an intersection point to the final destination nodes around it within a $3 \mathrm{Km} \times 3 \mathrm{Km}$ area.

Table 2: Values of $T_{\text {wireless }}$ with $\lambda_{\text {download }} / \mu_{\text {download }}=0.8$ for different uplink and downlink speeds

\begin{tabular}{|c|l|c|c|c|}
\hline \multicolumn{3}{|c|}{ Values of $T_{\text {wireless }}($ in $\mu \mathrm{s})$} \\
\hline $\begin{array}{c}\text { Downlink speed } \\
\text { (Mbps) }\end{array}$ & \multicolumn{4}{|c|}{ Uplink speed } \\
\cline { 2 - 6 } & 10 & 20 & 30 & 50 \\
\hline 100 & 1296 & 896 & 766 & 656 \\
\hline 200 & 1056 & 656 & 526 & 416 \\
\hline 500 & 912 & 512 & 382 & 276 \\
\hline 1000 & 864 & 464 & 334 & 224 \\
\hline
\end{tabular}

With these values, assuming an uplink speed of $50 \mathrm{Mbps}$ and a downlink speed of $1 \mathrm{Gbps}$, the overall end-to-end average latency for communicating a packet from $S$ to $D$ comes out to be $(88.1628+224) \mu \mathrm{s}=312.1628 \mu \mathrm{s}$.

\section{Extension to Rectangular Grid Backbone Network}

It is straightforward to extend our proposed approach to the network architecture with a general $M \times N$ rectangular grid structure for the optical fiber backbone network.

Let us denote $\min (M, N)$ by $\rho$ and $\max (M, N)$ by $\tau$. The number of intersection points on a transmission front $W_{j+1}$ will be one more than that on $W_{j}$ for $1 \leq j<\rho$, and will be one less than that on $W_{j}$ for $\tau \leq j<M+N-1$. For these two cases, to 
minimize the average queuing delay at $W_{j+1}$, the optimal fractions of mailbags to be routed along the up/right link from an intersection point $I_{i j}$ on $W_{j}$ will follow the results given in Theorems 1 and 2 , respectiveely, of [23].

For $\rho \leq j<\tau$, we need to consider one additional phase, which is termed as (unchanging phase), for routing mailbags in an optimal way from the intersection points on the transmission front $W_{j}$ to those on $W_{j+1}$. For this, we consider two cases as given below.

Case $1: M>N$.. In this case, the optimal values of $f_{i j}$ 's will follow directly from the results given in Theorem 3 of [23].

Case $2: M<N$. In this case, the optimal values of $f_{i j}$ 's will follow directly from the results given in Theorem 4 of [23].

\section{$7 \quad$ Multiple Destination Points}

All the previous results pertain to a grid backbone network with a single destination situated in the upper rightmost corner of the grid. We now consider a more generalized scenario where any intersection-point on an $M \times N$ grid can be the destination of some mailbags. According to our previous assumptions, mailbags are being transmitted through either right link or up link and a queue is formed at every intersection-point. But in this scenario of multiple destination points, depending on the relative positions of the source and destination transmission-fronts, mailbags can be transmitted through any of the four existing links - right link, up link, left link and down link from an intersection point. To tackle such a situation, two queues at each intersection point are used - i) a forward queue as described in the previous sections to be formed by the mailbags directed towards right link or up link and ii) a backward queue formed by the mailbags to be directed towards left link or down link. As discussed in Sections 3.1 and 3.2, we would optimize the queuing delay in both the forward and backward queues independently and obtain the corresponding optimal load distribution fractions from the two queues at each intersection point by suitably modifying our above results for a single destination point.

Let $W_{\sigma}$ and $W_{\delta}$ be the transmission-fronts containing the source node $I_{s \sigma}$ and the destination node $I_{d \delta}$, respectively, of a specific mailbag. Depending on the values of $s, \sigma, d$ and $\delta$ three different cases are considered as given below.

- Case 1: For $\sigma<\delta$, mailbags will be transmitted toward the top-right-most corner of the rectangular grid through the forward queue. From any intersection point, the mailbags are transmitted through the right/up link, following the optimal traffic distribution fractions depending on the total traffic on the forward queue. Optimal traffic distribution fractions will be computed using the same results as given in previous section, with some minor modifications as explained below. 
- Case 2: For $\sigma>\delta$, mailbags will be transmitted towards the bottommost leftmost corner point through the backward queue. From any intersection point, the mailbags are transmitted through the left/down link, following the optimal traffic distribution fractions depending on the total traffic on the backward queue. Optimal traffic distribution fractions will be computed by using the same results as for the forward queue, but with some appropriate mapping technique.

- Case 3: For $\sigma=\delta$, mailbags will be transmitted through the backward queue if $d>s$; otherwise mailbags will be transmitted through the forward queue.

\subsection{Actions on Mailbags in Forward Queues}

A mailbag in a forward queue will follow the optimal distribution fractions as given in Theorems 1, 2, 3 and 4 [23] with the following exceptions. If a mailbag is found to arrive at an intersection point which is already in the same row (column) as that of its final destination, then to avoid any detour, we do not distribute the mailbags depending on the optimal traffic distribution fractions computed following the results of Theorems 1,2, 3 and 4 [23]; rather we push that mailbag along that particular row/column to reach its destination. Also, while a mailbag is transmitted from one transmission-front to the next to reach an intermediate intersection point $I_{i \delta}$ which lies on the same transmission-front of its destination at, say, $I_{d \delta}$, then this mailbag will be shifted to the backward queue for $d>i$ (similar to initial disposition according to Case 3 above) for subsequently forwarding it towards the destination.

\subsection{Actions on Mailbags in Backward Queues}

For the backward queues, it is first needed to determine the optimal-distribution fractions of the mailbags at any intersection point. To do this, we first assume that all the mailbags in the backward queues have the same (single) destination point at the bottommost leftmost corner of the grid so that at an intersection point, a mailbag will always be routed along the left link or the down link. For computing the optimal-distribution fractions for the mailbags in the backward queue with such a single destination point at one corner-point of the grid, we use the results from Theorems 1, 2, 3 and 4 [23] for the forward queues with a suitable mapping of the transmission-fronts and the intersection points. For this mapping, let us assume an imaginary line connecting the topmost rightmost corner point and the diagonally opposite bottommost leftmost corner-point. Let us then draw lines perpendicular to this imaginary line, each passing through the grid points $W_{1}^{\prime}, W_{2}^{\prime}, \cdots$ in Fig. 5 (depicted by the dotted lines), which we identify as the transmissionfronts of the backward queues. For the backward queues, the intersection points on a transmission-front will be numbered as in Fig. 5. Total number of intersection points over each of these transmission-fronts $W_{1}^{\prime}, W_{2}^{\prime}, \cdots$ is calculated in a similar 
way as that for the forward queues. If the same grid point is denoted by $I_{i j}$ and $I_{q r}^{\prime}$ corresponding to the forward queues and backward queues, respectively, then the mapping between $I_{i j}$ and $I_{q r}^{\prime}$ is defined as follows, where $\min (M, N)$ is denoted by $\rho$ and $\max (M, N)$ by $\tau$.

$$
I_{i j}= \begin{cases}I_{(j-i+1),(M+N-j)}^{\prime}, & \text { for } 1 \leq j<\rho \\ I_{(\rho-i+1),(M+N-j)}^{\prime}, & \text { for } \rho \leq j \leq \tau \\ I_{(M+N-j-i+1),(M+N-j)}^{\prime}, & \text { for } \tau<j \leq M+N-1\end{cases}
$$

That is,

$$
(q, r)= \begin{cases}(j-i+1, M+N-j), & \text { for } 1 \leq j<\rho \\ (\rho-i+1, M+N-j), & \text { for } \rho \leq j \leq \tau \\ (M+N-j-i+1, M+N-j), & \text { for } \tau<j \leq M+N-1\end{cases}
$$

After this mapping, the results obtained in TTheorems 1, 2, 3 and 4 [23] can be directly applied for the forward queues to obtain the optimal distribution fractions of mailbags between $W_{j}^{\prime}$ and $W_{j+1}^{\prime}$ corresponding to the backward queues and only one destination point at the bottommost leftmost corner of the grid.

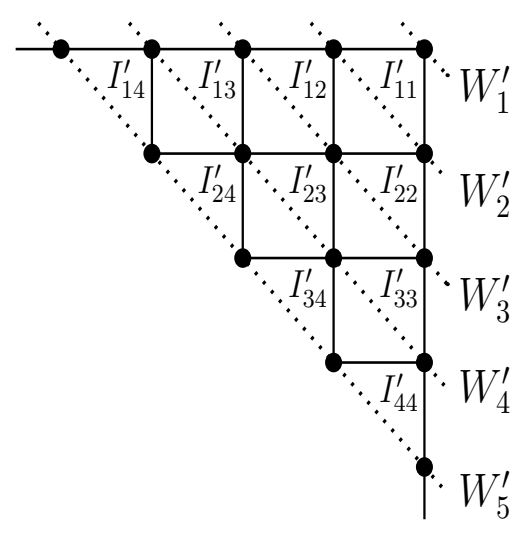

Fig. 5: Transmission-fronts and intersection points in backward queue

For the multiple destination points, we propose to use the same technique as discussed above for the mailbags in the forward queue. That is, we normally use the optimal distribution fractions as computed above for transferring a mailbag in a backward queue from $W_{j}^{\prime}$ to $W_{j+1}^{\prime}$, with the following exception. If a mailbag is found to already arrive at an intersection point which is in the same row (column) as that of its final destination, then to avoid any detour, we do not distribute the mailbags depending on the optimal traffic distribution fractions computed above; 
rather we push that mailbag along that particular row/column to reach its destination. Also, while a mailbag is transmitted from one transmission-front to the next to reach an intermediate intersection point $I_{i j}$ which lies on the same transmissionfront of its destination at, say, $I_{d j}$, then this mailbag will be shifted from the backward queue to the forward queue for $d<i$ (just like initial disposition of mailbags as in Case 3 above) for subsequently forwarding it towards the destination.

\subsection{Routing a Mailbag}

Assume that a mailbag $\mathcal{M}$ is formed at a source intersection point $I_{s \sigma}$ whose final destination point is $I_{d \delta}$. The algorithm used for placing $\mathcal{M}$ in the appropriate queue and subsequent routing along the grid is described below in Algorithm 1.

\section{Simulation Results}

The result of simulating Algorithm 1 using an ns-3 simulator is now presented. We simulate a 500 Gbps optical fiber grid network of dimension $6 \times 6$ as the backbone laid over a $15 \mathrm{Km} \times 15 \mathrm{Km}$ city area and a $100 \mathrm{Mbps}$ wireless communication channel between source (or, destination) and the access point of the backbone optical network. The experiment can, however, be easily extended to a grid of higher dimension covering a much larger metropolitan area. A total number of 1,000,000 mailbags has been generated all over the grid. Coordinates of the destination points for each mailbag has been generated in a random manner. Fig. 6 shows the results of this simulation with packets each of size $1 \mathrm{~KB}$ generated with the sum total of all $\lambda$ values over all intersection points varied from 0.65 to 0.9 .

From Fig. 6 we can observe that the average routing time for individual packets varies from 46-79 $\mu$ s over the backbone network. We also simulate the queue of received packets at the receive buffer of the destination intersection point on the backbone network and find an average value of $T_{\text {wireless }}$ ranging from 244 to $301 \mu \mathrm{s}$, by varying $\lambda_{\text {download }} / \mu_{\text {download }}$ from 0.6 to 0.9 , corresponding to an uplink speed of $50 \mathrm{Mbps}$ and downlink speed of $1 \mathrm{Gbps}$. Thus, the overall end-to-end latency comes out to be less than $(79+301)=380 \mu$ s using 50 Mbps uplink speed and 1 Gbps downlink speed.

\section{Conclusion}

A dramatic reduction of end-to-end latency in message communication to meet the requirements of future applications will be one of the major drivers for the transition from $5 \mathrm{G}$ to $6 \mathrm{G}$ communication networks. In view of that, we have proposed in this paper a novel approach for data routing in a $6 \mathrm{G}$ WAN that involves a paradigm shift from existing packet routing strategies. Our proposed approach is 


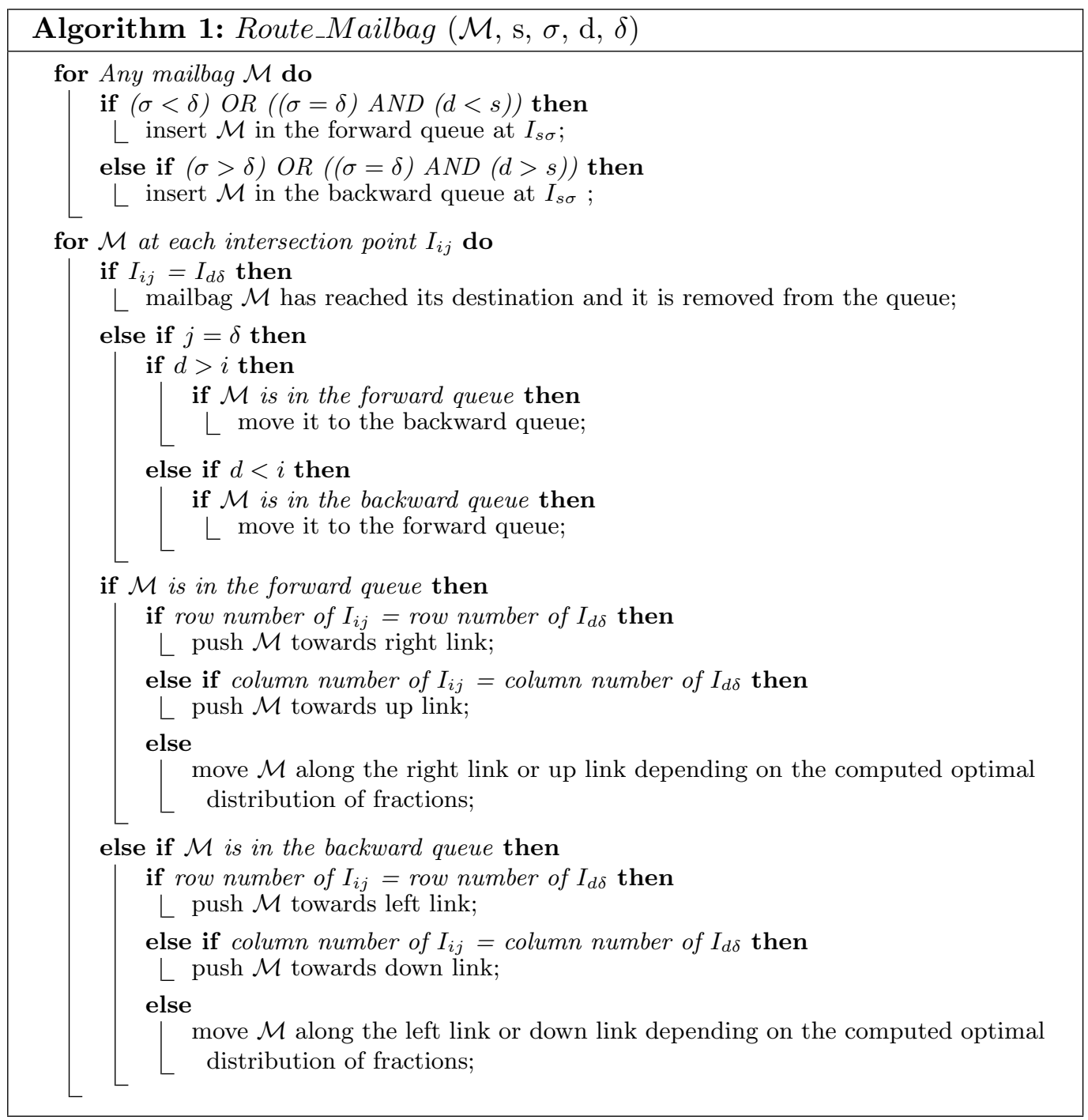




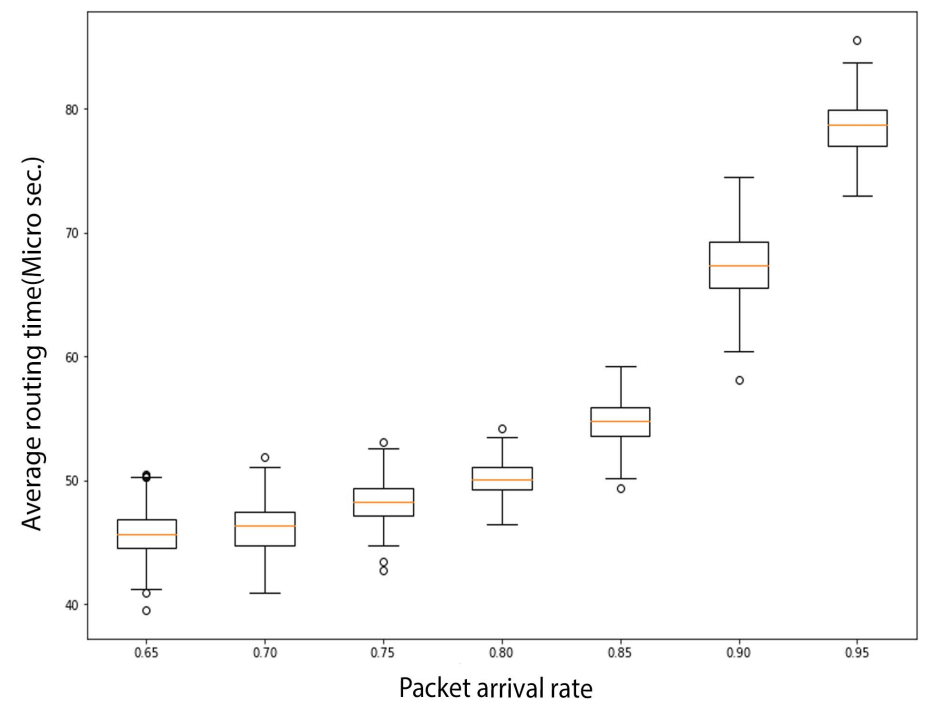

Fig. 6: Average routing time considering all the packets in the network

based on mimicking how road networks are designed for large cities. Towards this goal, we assume that the backbone network of the WAN is built using high-speed optical fibers. Messages from a source node are routed to the nearest access point on this backbone network through a local wireless network. The backbone network then delivers the message with very low latency to some access point nearest to the destination node. Finally the message reaches its desired destination from this access point through a local wireless network. In order to significantly reduce the message processing overhead in intermediate routers, multiple messages are coalesced together to form a mailbag. Next, extension of this technique to a optical, rectangular grid network as the backbone and having multiple destination points at any location across the grid has been presented. Our proposed approach has been simulated using ns-3 for a 500 Gbps optical fiber grid network as the backbone laid over a $15 \mathrm{Km} \times 15 \mathrm{Km}$ city area and a wireless communication channel having 50 Mbps uplink speed from the source and 1000 Mbps downlink speed to the destination. The simulation results show that the average end-to-end latency for a packet of size $1 \mathrm{~KB}$ is less than $380 \mu \mathrm{s}$, while the time taken to route packets through the backbone network requires only 46-79 $\mu$ s under varying traffic conditions. Given the observed ultra-low communication latencies in our ns-3 simulations, our results thus clearly demonstrate the potential of our proposed routing strategy for meeting the ultra-low latency requirements of current $5 \mathrm{G}$ and future $6 \mathrm{G}$ networks. Future research in this direction may include replacing the Manhattan grid network in the backbone by concentric rings of optical fibers and distributing the message traffic 
for optimal queuing delay at the intermediate routers, and also system level implementation of the proposed idea using a prototype architecture.

\section{Conflicts of Interest}

The authors declare no conflict of interest.

\section{References}

1. Tum. https://www.technologist.eu/new-record-data-transfer-speed-in-fiber-optic-network/. Accessed: 2020-03-09.

2. N. Abbas, Y. Zhang, A. Taherkordi, and T. Skeie. Mobile edge computing: A survey. IEEE Internet of Things Journal, 5(1):450-465, 2018. doi: 10.1109/JIOT.2017.2750180.

3. M. K. Abdel-Aziz, S. Samarakoon, M. Bennis, and W. Saad. Ultra-reliable and low-latency vehicular communication: An active learning approach. IEEE Communications Letters, 24(2):367-370, 2020. doi: 10.1109/LCOMM.2019.2956929.

4. A. Al-Fuqaha, M. Guizani, M. Mohammadi, M. Aledhari, and M. Ayyash. Internet of things: A survey on enabling technologies, protocols, and applications. IEEE Communications Surveys Tutorials, 17(4):2347-2376, 2015. doi: 10.1109/COMST.2015.2444095.

5. J. N. Al-Karaki and A. E. Kamal. Routing techniques in wireless sensor networks: a survey. IEEE Wireless Communications, 11(6):6-28, 2004. doi: 10.1109/MWC.2004.1368893.

6. F. Alassery and M. M. Althobaiti. Context information aggregation mechanism based on bloom filters (cia-bf) for high performance monitoring applications of internet of things. International Journal of Computer Networks \& Communications (IJCNC), 13(1), Jan. 2021. doi: 10.5121/ijcnc.2021.13107.

7. J. G. Andrews, S. Buzzi, W. Choi, S. V. Hanly, A. Lozano, A. C. K. Soong, and J. C. Zhang. What will $5 \mathrm{~g}$ be? IEEE Journal on Selected Areas in Communications, 32(6):1065-1082, 2014. doi: 10.1109/JSAC.2014.2328098.

8. L. Bariah, L. Mohjazi, S. Muhaidat, P. Sofotasios, G. Karabulut Kurt, and O. Dobre. A prospective look: Key enabling technologies, applications and open research topics in $6 \mathrm{~g}$ networks. IEEE Access, PP:1-1, 08 2020. doi: 10.1109/ACCESS.2020.3019590.

9. S. Buzzi, C. I, T. E. Klein, H. V. Poor, C. Yang, and A. Zappone. A survey of energyefficient techniques for $5 \mathrm{~g}$ networks and challenges ahead. IEEE Journal on Selected Areas in Communications, 34(4):697-709, 2016. doi: 10.1109/JSAC.2016.2550338.

10. J. Cho and P. J. Winzer. Probabilistic constellation shaping for optical fiber communications. Journal of Lightwave Technology, 37(6):1590-1607, 2019. doi: 10.1109/JLT.2019.2898855.

11. K. David and H. Berndt. $6 \mathrm{~g}$ vision and requirements: Is there any need for beyond $5 \mathrm{~g}$ ? IEEE Vehicular Technology Magazine, 13(3):72-80, 2018. doi: 10.1109/MVT.2018.2848498.

12. D. Ferguson, A. Lindem, and J. Moy. OSPF for IPv6. RFC 5340, July 2008. doi: $10.17487 / \mathrm{RFC} 5340$.

13. W. Heinzelman, A. Chandrakasan, and H. Balakrishnan. Energy-efficient communication protocol for wireless microsensor networks. In Proceedings of the 33rd Annual Hawaii International Conference on System Sciences, page 10 pp. vol.2, 2000. doi: 10.1109/HICSS.2000.926982.

14. M. Jammal, T. Singh, A. Shami, R. Asal, and Y. Li. Software-defined networking: State of the art and research challenges. Computer Networks, 72, 05 2014. doi: 10.1016/j.comnet.2014.07.004.

15. M. Maier. Fiber-wireless (fiwi) broadband access networks in an age of convergence: Past, present, and future. Advances in Optics, 2014:1-23, 06 2014. doi: 10.1155/2014/945364.

16. G. S. Malkin. RIP Version 2. RFC 2453, Nov. 1998. 
17. J. Moy. Rfc2328: Ospf version 2, April 1998. doi: 10.17487/RFC2328.

18. S. Panwar. Breaking the millisecond barrier: Robots and self-driving cars will need completely reengineered networks. IEEE Spectrum, 57(11):44-49, 2020. doi: 10.1109/MSPEC.2020.9262144.

19. P. Popovski, K. F. Trillingsgaard, O. Simeone, and G. Durisi. 5g wireless network slicing for embb, urllc, and mmtc: A communication-theoretic view. IEEE Access, 6:55765-55779, 2018. doi: 10.1109/ACCESS.2018.2872781.

20. V. Raghavan and J. Li. Evolution of physical-layer communications research in the post-5g era. IEEE Access, pages 10392-10401, 2019. doi: 10.1109/ACCESS.2019.2891218.

21. Y. Rekhter, S. Hares, and T. Li. A Border Gateway Protocol 4 (BGP-4). RFC 4271, Jan. 2006. doi: 10.17487/RFC4271.

22. J. Ren, D. Zhang, S. He, Y. Zhang, and T. Li. A survey on end-edge-cloud orchestrated network computing paradigms: Transparent computing, mobile edge computing, fog computing, and cloudlet. ACM Computing Surveys, 52:1-36, 10 2019. doi: 10.1145/3362031.

23. S. S. Sarma, K. Sinha, C. Sub-r-pa, G. Chakraborty, and B. P. Sinha. Optimal distribution of traffic in manhattan road networks for minimizing routing-time. IEEE Transactions on Intelligent Transportation Systems, pages 1-22, 2020. doi: 10.1109/TITS.2020.2994836.

24. F. Tariq, M. R. A. Khandaker, K. K. Wong, M. A. Imran, M. Bennis, and M. Debbah. A speculative study on 6g. IEEE Wireless Communications, 27(4):118-125, 2020. doi: 10.1109/MWC.001.1900488.

25. D. ThanhChuong, H. L. Cuong, P. T. Duc, and D. D. Hung. Performance analysis in cellular networks considering the QoS by retrial queueing model with the fractional guard channels policies. International Journal of Computer Networks $\&$ Communications (IJCNC), 13(4), Jul. 2021. doi: 10.5121/ijcnc.2021.13406.

26. Y. Tsuyoshi and F. Satoshi. Towards fog-assisted virtual reality mmog with ultra-low latency. International Journal of Computer Networks \& Communications (IJCNC), 12(6), Nov. 2020. doi:10.5121/ijcnc.2020.12603.

27. H. Win and A.-S. Pathan. On the issues and challenges of fiber-wireless (fi-wi) networks. Journal of Engineering, 2013, 04 2013. doi: 10.1155/2013/645745.

28. P. Yang, Y. Xiao, M. Xiao, and S. Li. $6 \mathrm{~g}$ wireless communications: Vision and potential techniques. IEEE Network, 33(4):70-75, 2019. doi: 10.1109/MNET.2019.1800418.

29. S. Zhang, C. Xiang, and S. Xu. 6g: Connecting everything by 1000 times price reduction. IEEE Open Journal of Vehicular Technology, 1:107-115, 2020. doi: 10.1109/OJVT.2020.2980003.

30. Z. Zhao, C. Feng, H. H. Yang, and X. Luo. Federated-learning-enabled intelligent fog radio access networks: Fundamental theory, key techniques, and future trends. IEEE Wireless Communications, 27(2):22-28, 2020. doi: 10.1109/MWC.001.1900370.

\section{Authors}

Satya Ranjan Das received his M.Tech. degree in Information Technology from IIT, Kharagpur, India in 2011. He is currently pursuing his Ph.D. at Jadavpur University, Kolkata, India and also working as an Assistant Professor in the department of Computer Science and Engineering, S'O'A University, Bhubaneswar, 
India.

Sayan Sen Sarma received his M.Tech degree in Computer Science \& Engg. from University of Calcutta, Kolkata, India, in 2014. Presently, he is pursuing his PhD at Department of Computer Science and Engineering, University of Calcutta, Kolkata, India and also working as an Assistant Professor in Department of Compute Science, Mahishadal Girls' College, West Bengal, India.

Mitrabinda Khuntia received her M.Tech degree from Institute of Technical Education \& Research (ITER), Siksha 'O' Anusandhan University in 2011. Presently, she is working as an Assistant Professor in the Department of Computer Science and Engineering, ITER, Siksha 'O' Anusandhan University.

Indranil Roy is currently a $\mathrm{PhD}$ student in the School of Computing, Southern Illinois University, Carbondale. He completed his MS in Computer Science from SIU, Carbondale in 2018.

Koushik Sinha is an Associate Professor in the School of Computing, Southern Illinois University, Carbondale, USA. He received the Indian Science Congress Young Scientist Award for 2008-2009 and N. V. Gadadhar Memorial Award from the Institution of Electronics and Telecommunication Engineers, India in 2011. He is a senior member of IEEE, USA and a member of the ACM.

Bhabani P. Sinha served as a faculty member in the Indian Statistical Institute, Kolkata, India from 1976 to 2017. He is currently a Distinguished Professor in the Dept. of Computer Science \& Engg. at SOA University, Bhubaneswar. He is a Fellow of the IEEE, USA. 